but simply by gravity. The fact that the addition of salt reduces the viscosity of the fresh latex very considerably is one of the reasons for this.

The true nature of the viscous matter in fresh latex is not yet known, but the properties resemble those of coacervates ${ }^{3}$ very much; thus it seems probable that fresh latex contains a micro-coacervate which by centrifuging or under favourable conditions as to the viscosity goes over in a macro-coacervate.

The progress of our research work has been slowed down, first by the War and later it was interrupted by the unhappy conditions in Java; it is expected, however, that work at our Institute will be resumed in the near future.

${ }^{1}$ Compare v. Gils, Arch. Rubbercultuur, 25, 395 (1941).

Symontowne, Ind. Rubb. W.108.148259 (1943).

' See Kruyt and Bungenberg de Jong, Koll. Z.. 50, 39 (1930).

\section{STUDIES IN STOMATAL ACTION}

\section{Adequacy of the Porometer in the Investigation of Stomatal Aperture}

$I^{\mathrm{N}}$ the usual form of porometer a small cup is permanently attached to the leaf surface, air is drawn through the leaf into the cup under reduced pressure and either the rate of air flow is measured ${ }^{1,2}$ or, in the 'resistance porometer' 3,4 , the fall in pressure across the leaf and also that across a standard capillary resistance in series is found; from these pressure gradients the resistance to flow through the leaf is calculated. The rate of flow or the resistance to flow are functions, though very complex ones, of stomatal aperture. In earlier work porometers were used for the purely qualitative purpose of showing whether the stomata were opening or closing. Interest in the dynamics of stomatal movement $t^{5,6}$ and in the relation between stomatal movement and carbon assimilation ${ }^{2,8}$, however, made it desirable to attempt to estimate from porometer readings the resistance to viscous flow of the stomata alone, thus eliminating the confusing effects of the irrelevant internal resistances in the leaf. Hence, ultimately, making use of the less convenient 'diffusion porometer' ${ }^{\prime}$ to calibrate the 'resistance porometer', the resistance to diffusion through the stomata might be estimated. With this end in view a mathematical theory of porometers was elaborated ${ }^{10,4}$ and used to estimate stomatal resistances in Pelargonium 4 .

During a recent investigation of shock-induced stomatal movements ${ }^{11}$, it was found that with stomata open in the light, strips of epidermis taken by Lloyd's ${ }^{12}$ method within the cup area showed very much wider apertures than those taken just outside the cup. This was at first attributed to the lack of shock sensitivity of the stomata within the cup ${ }^{11}$. The possibility existed, however, that the stomata outside the cup were not showing 'shock closure' on stripping of the epidermis, but actually had smaller apertures on the intact leaf than those within the cup. This, if true, would have an important bearing on the quantitative use of porometers, for the theory mentioned above demands the assumption that the stomatal apertures are the same inside and outside the cup.

A considerable number of experiments, carried out separately after consultation between us, has left no doubt that illuminated leaves, both of Pelargonium zonale and Triticum vulgare, have, in fact, much wider stomatal apertures where the surface is enclosed in a cup than elsewhere. This has been shown by a modification of Molisch's ${ }^{13}$ injection technique using gentian violet in absolute alcohol (Pelargonium and Triticum) as well as by Lloyd's method and direct microscopical observation (Pelargonium). If part of the lamina of a Pelargonium or wheat leaf is merely placed between two glass strips and then, after two or three hours illumination, flooded with alcohol, heavy injection indicating wide open stomata is found where the leaf has been enclosed, and little or none elsewhere. Darkened portions show no injection either within or outside the enclosed area. Similar results are obtained using small chambers resembling porometer cups.

The conditions within a porometer cup differ from those outside in the following respects: (1) The air inside the cup is of restricted volume and at rest between readings (if the porometer is disconnected); it thus rapidly becomes saturated with water vapour, a process which is hastened by the drawing of air from the intercellular space system of the leaf into the cup during readings. In the case of Pelargonium, the concentration of ethereal oils must also be higher within the cup; with any species other gaseous substances may diffuse out of the leaf, and the oxygen and carbon dioxide tensions in the cup will also be affected. (2) The upper surface of the leaf above the cup is (normally) touching a glass plate. (3) The temperature within the cup may be slightly higher. (4) The leaf in the cup area is (usually) illuminated from above only, the cup shading the lower surface from any direct light.

The possible effects of these differences may now be considered.

(1) Experiments, each witn a pair of porometer cups on the same leaf, one cup containing a desiccating agent (silica gel or calcium chloride) and the other empty or with moist broken porous tile, show similar wide stomatal opening in both cups, whether judged by porometer readings or Lloyd's method. These results seem to dispose of high humidity as the factor causing the wide opening within the cup. The ethereal oils of Pelargonium also seem unlikely to be important in this respect, for similar effects are found with wheat. The question of other variations in the composition of the air in the cup is discussed elsewhere ${ }^{14}$.

(2) It is found that similar wide stomatal opening occurs when Pelargonium or wheat plants are enclosed in a bell jar, in a saturated atmosphere, with the leaves not in contact with the glass. Thus pressure of the glass plate on the leaf is not concerned. Similar plants outside the bell jar show little opening except where the leaves are enclosed between glass plates, as above.

(3) In the bell jar experiments just cited the difference in temperature inside and outside does not exceed $1 \frac{1}{2}^{\circ} \mathrm{C}$. Thus the differences in aperture can arise in the absence of appreciable temperature differences.

(4) These experiments have been carried out with moderate light intensities-a 100-W. or 150-W. lamp, with running water screen, at 3-7 in. distance. It seems improbable that reducing the illumination of the lower surface of the leaf would cause wider stomatal opening. In all cases the usual closing response to darkening the upper surface has been found.

Whatever the cause of the wider opening within the cup, and this is the subject of a further com- 
munication from one of us $^{14}$, it is essential to overcome it if the porometer is to be used other than to show in a purely qualitative manner whether the stomata are opening or closing. The ideal to aim at is obviously to make the conditions within the cup the same as those outside. This has been to a large extent achieved by a simple modification of the form of resistance porometer used by Heath ${ }^{4}$. Air of the same composition and humidity as that surrounding the leaf is supplied to the porometer at a constant increased pressure by a simple aspirator working on the 'gasometer' principle. To avoid alteration of the humidity, medicinal paraffin is used in the aspirator and a light grade of the same in the manometers. As compared with the normal type of resistance porometer the pressure gradients are all reversed, but the manometer readings are used in the usual way, only the signs being changed. A hole in the glass or 'Perspex' plate pressing the leaf against the cup washer leaves all the upper leaf surface above the cup area open to the air. Between readings the cup is swept with a stream of the same air that surrounds the rest of the leaf, and during readings such air is being forced from the cup into the leaf through the stomata. There is therefore no tendency for water vapour or other gaseous substances from the leaf to accumulate in the cup. Using this modification of the porometer, preliminary tests with Pelargonium have indicated that the stomal apertures inside and outside the cup, as shown by Lloyd's method, are of similar magnitude. A preferable alternative to sweeping air through the cup would be the use of a detachable cup. Maskell?, using the smooth-leafed cherry laurel, was able to use a cup with a gelatine washer which was simply pushed against the leaf and could be completely removed between readings. For hairy leaves such as Pelargonium, however, it is necessary to use a special design of detachable cup which leaves the washer in position on the leaf between readings. Such cups have been used previously for Pelargonium in the Research Institute of Plant Physiology, and the method is at present in use in experiments with wheat. A similar cup will now be tried with Pelargonium, which is easier than wheat to examine by Lloyd's method, to see whether it gives similar apertures within and outside the cup area.

It is likely that with readings of short duration a normal (reduced pressure) resistance porometer might safely be used; but the modification described above reduces the risk and does not appear to have any disadvantages. The 'gasometer' type of aspirator, which can, of course, be used for either increased or reduced pressures, has the important advantage over the usual type that there is no bubbling of the air through a liquid and hence the menisci in the manometers are absolutely steady and readings can be made instantaneously.

It should be emphasized that data obtained with the more usual type of porometer and a permanently fixed unswept cup are valid for the enclosed stomata so far as qualitative indication of opening or closure in response to light or darkness is concerned. The stomata within the cup do respond to light, and open much wider than those outside. It may be noted also that when used with double 'leaf chambers's, in which a considerable area outside the porometer cup is maintained under identical conditions, the normal type of porometer yields data which as an approximation at least may be treated quantitatively in the manner indicated elsewhere 4 .
This work is continuing and will be published in detail in due course.

Research Institute of Plant Physiology, O. V. S. Heath

Imperial College, London, S.W.7.

$$
\text { W. T. WrLIIAMs }
$$

Botany Department,

Bedford College for Women, London, N.W.1.

${ }^{1}$ Darwin, F., and Pertz, D. F. M., Proc. Roy. Soc., B, 84, 136 (1911). ' Knight, R. C., New Phyt., 14, 212 (1915).

- Gregory, F. G., and Pearse, H. L., Proc. Roy. Soc., B, 114, 477 (1934).

'Heath, O. V. S., Ann. Bot., N.S., 5, 455 (1941).

-Gregory, F. G., and Pearse, H. L., Ann. Bot., N.S., 1, 3 (1937).

- Williams, W. T., Ph.D. Thesis, Univ. Lond. (1940).

' Maskell, E. J., Proc. Roy. Soc., B, 102, 488 (1928).

Beath, O. V. S., Ann. Bot., N.S., 3, 469 (1939).

- Gregory F. G., and Armstrong, J. I., Proc. Roy. Soc., B, 121, 27 (1936).

${ }^{10}$ Penman, H. L., Proc. Roy. Soc., B, 139, 416 (1942).

11 Williams, W. T., Nature, 160, 364 (1947).

${ }^{12}$ Lloyd, F. E., Pub. Carneg. Inst., 82 (1908).

${ }_{13}$ Molisch, H., Z. Bot., 4, 106 (1912).

14 Heath, O. V. S., following communication.

\section{Control of Stomatal Movement by a Reduction in the Normal Carbon Dioxide Content of the Air}

To account for the much wider stomatal apertures found within a porometer cup than elsewhere on an illuminated leaf ${ }^{1}$, the hypothesis was examined that some volatile substance produced by the leaf itself caused opening of the stomata in light when in high concentration. On testing this hypothesis a considerable amount of experimental evidence was obtained in its favour. Thus detached leaves in water of Pelargonium or wheat, enclosed together with entire plants in a saturated atmosphere under a bell jar and illuminated for a few hours, showed wide stomatal opening when tested by the injection technique of Williams ${ }^{1}$; single leaves similarly enclosed by themselves, also in a saturated atmosphere and illuminated, showed little or no injection. On the other hand, similar single leaves of wheat each enclosed in a small volume of saturated air in a glass tube showed very heavy injection, as in the leaves from the crowded bell jars. When such tubes were swept continuously with a slow stream of humid air, little or no injection of the leaves was found.

The following statistically significant effects were thus obtained : (1) Heavier injection (wider stomatal opening) with many than with few or single leaves in the same large volume of humid still air ; (2) heavier injection with single leaves in a small than in a large volume of humid still air ; (3) heavier injection with single leaves in a small volume of humid still air than in humid moving air. Porometer experiments with Pelargonium showed that sweeping the cup with moving air, whether dry or humid, caused considerable stomatal closure as compared with still air. Similar results have been obtained with wheat, using dry air, by Mr. Milthorpe in this Institute. Darwin's finding that removal of plants from a (presumably crowded) greenhouse to the laboratory caused stomatal closure, but that enclosure of the plants in bell jars caused the stomata to remain open or re. open, also seemed to support the hypothesis, although he himself attributed these effects to humidity changes.

There existed, however, the seemingly remote possibilities that all these effects were due either to 